\title{
EXAMPLES OF VARIETIES WITH INDEX ONE ON $C_{1}$-FIELDS
}

\author{
ANANYO DAN AND INDER KAUR
}

\begin{abstract}
Let $K$ be the fraction field of a Henselian discrete valuation ring with algebraically closed residue field $k$. In this article we give a sufficient criterion for a projective variety over such a field to have index 1 .
\end{abstract}

\section{INTRODUCTION}

A field $K$ is called $C_{1}$ if any degree $d$ polynomial in $n$ variables with $n>d$ has a nontrivial solution. The $C_{1}$ conjecture due to Lang, Manin and Kollár states that every separably rationally connected variety over a $C_{1}$ field has a rational point. The conjecture has already been proven for several $C_{1}$-fields (see [Kau16] for a complete discussion). However it is still open in the case when $K$ is the fraction field of a Henselian discrete valuation ring of characteristic 0 with algebraically closed residue field of characteristic $p>0$. Recently, the conjecture was shown to hold trivially for certain rationally connected varieties over such fields (see [Kau18]).

It is natural to ask whether a similar conjecture holds if we replace the condition for a rational point by the condition of index one and weaken the condition on rational connectedness. Recall that the index of a variety $X$, denoted ind $(X)$, is the gcd of the set of degrees of zero dimensional cycles on $X$. In [ELW15, Corollary 2.5], Esnault, Levine and Wittenberg prove that if $X$ is a smooth, projective variety over the fraction field of a Henselian discrete valuation ring with algebraically closed residue field of characteristic 0 , then $\operatorname{ind}(X)$ divides the Euler characteristic of the structure sheaf of $X$. Using this they prove that, in the case $X$ is a rationally connected variety over such a field, we have $\operatorname{ind}(X)=1$ (see [ELW15, Corollary 3]). Since the Euler characteristic of $\mathcal{O}_{X}$ is one if and only if $X$ has arithmetic genus 0 , this gives a positive answer to the modified conjecture only for very few choices of $X$.

In this article we study a weaker notion of index, which we call the linear index. Let $K$ be a field of characteristic zero and $X$ a projective $K$-variety. We define the linear index of $X$, denoted $\operatorname{ind}_{\text {lin }}(X)$, to be the gcd of the Euler characteristic of the set of line bundles on $X$. The definition of linear index is inspired by Kollár's definition of elw-index, denoted elw $(X)$, as given in Kol13, which is the ged of the Euler characteristic of all coherent sheaves on $X$. An advantage of using $\operatorname{ind}_{\operatorname{lin}}(X)$ over $\operatorname{elw}(X)$ is that it is much easier to compute the index of $X$ using the former notion. In particular, it is extremely hard to enumerate the set of all coherent sheaves on a variety (even fixing Hilbert polynomial is not sufficient to guarantee boundedness of families of coherent sheaves, see [HL10]). In comparison, the set of all invertible sheaves is given by the Picard group, which is of finite rank in numerous examples. Moreover, the Euler characteristic of an invertible sheaf is significantly easier to compute than that of a general coherent sheaf (see Riemann Roch formula for coherent sheaves [BFM75]). As a result, we are able to give a simple combinatorial criterion under which a variety has index 1 . More precisely,

Date: January 7, 2020.

2010 Mathematics Subject Classification. Primary 12G05, 16K50, 14D20, 14J60, Secondary 14L24, 14 D22.

Key words and phrases. Index of varieties, $C_{1}$-fields, geometrically stable sheaves, Galois descent, Galois cohomology, Brauer group. 
Theorem 1.1 (Theorem 2.7, Corollary 2.8). Fix an ample line bundle $H$ on $X$ such that if there exists an invertible sheaf $H_{0}$ on $X$ with $H_{0}^{\otimes n} \cong H$ then $n$ must be 1 or -1 . Suppose that $H^{1}\left(\mathcal{O}_{X}\right)=0, \operatorname{Pic}\left(X_{\bar{K}}\right)$ is of rank $r$, generated by $\mathcal{L}_{1}, \ldots, \mathcal{L}_{r-1}$ and $\mathcal{L}_{r}:=H_{\bar{K}}=H \otimes_{K} \bar{K}$ satisfying the following conditions:

(1) the ideal $\left(\operatorname{deg}\left(\mathcal{L}_{1}\right), \operatorname{deg}\left(\mathcal{L}_{2}\right), \ldots, \operatorname{deg}\left(\mathcal{L}_{r}\right)\right)$ in $\mathbb{Z}$ generated by $\operatorname{deg}\left(\mathcal{L}_{i}\right)$ for $i=1, \ldots, r$ coincides with the ideal (1), where degree of the invertible sheaves are taken with respect to $H_{\bar{K}}$ (see [HL10, Definition 1.2.11]),

(2) for any $r \times r$-matrix $A=\left(a_{i, j}\right)$ with integral entries $a_{i, j}, a_{r, k}=0$ for all $k<r, a_{r, r}=1$, $A \neq \operatorname{Id}$ and $A^{t}=\operatorname{Id}$ for some $t>0$, we have $\sum_{j} a_{i j} \operatorname{deg}\left(\mathcal{L}_{j}\right) \neq \operatorname{deg}\left(\mathcal{L}_{i}\right)$ for some $i>0$.

Then, each $\mathcal{L}_{i}$ is $G$-invariant and $\operatorname{gcd}\left\{\chi\left(\mathcal{L}_{i}(n)\right) \mid i=1, \ldots, r\right.$ and $\left.n \in \mathbb{Z}\right\}=1$. Moreover, if $K$ is a $C_{1}$-field, then $\operatorname{ind}_{\text {lin }}(X)=\operatorname{ind}(X)=1$ if $\operatorname{char}(k)=0$ and prime-to-p part of $\operatorname{ind}(X)$ and $\operatorname{ind}_{\text {lin }}(X)$ equals 1 if $\operatorname{char}(k)=p>0$.

By prime-to-p part of $N$ we mean the largest divisor of $N$ which is prime to $p$.

We use the criterion in Theorem 1.1 to give examples of non-rationally connected varieties having index one over a $C_{1}$-field (see Example 2.9 and Remark 2.10). In Example 2.11, we give examples in the case $K$ is not a $C_{1}$-field.

Acknowledgements The first author is supported by ERCEA Consolidator Grant 615655NMST and also by the Basque Government through the BERC 2014 - 2017 program and by Spanish Ministry of Economy and Competitiveness MINECO: BCAM Severo Ochoa excellence accreditation SEV-2013 - 0323. The second author is funded by a fellowship from CNPq Brazil. A part of this work was done when she was visiting ICTP. She warmly thanks ICTP, the Simons Associateship and Prof. C. Araujo for making this possible. We also thank the referee for several helpful suggestions.

\section{INDEX OF VARIETIES}

Notation 2.1. Let $K$ be a field of characteristic 0 and $X$ be a projective $K$-variety.

Definition 2.2. Given a smooth, quasi-projective $K$-variety $Y$, we define the associated (cohomological) Brauer group $\operatorname{Br}(Y)=H_{\text {ét }}^{2}\left(Y, \mathbb{G}_{m}\right)$.

Remark 2.3. If $K$ is the maximal unramified extension of a complete field, then $K$ is a $C_{1}$-field (see [Lan52]). Recall, for any $C_{1}$-field $K$, we have $\operatorname{Br}(K)=0$ (see [Ser13, §X.7]).

Remark 2.4. One can check the following elementary properties of $\operatorname{ind}_{\text {lin }}(X)$ :

(1) If $X$ is a projective $K$-curve containing a $K$-rational point, then $\operatorname{ind}_{\text {lin }}(X)=1$.

(2) This is not true in higher dimension. If $K=\mathbb{C}$ and $X$ is a very general smooth, projective quartic surface in $\mathbb{P}^{3}$ (by Noether-Lefschetz theorem, a very general quartic has Picard rank one), then $\operatorname{ind}_{\text {lin }}(X)$ is divisible by 2 (use Riemann-Roch theorem).

(3) For any $X$, $\operatorname{ind}_{\text {lin }}(X)$ divides the ged of the set of Euler characteristics of $H^{\otimes a}$ as $a$ varies over $\mathbb{Z}$. In particular, if $X$ is an odd degree surface in $\mathbb{P}_{K}^{3}$, then $\operatorname{ind}_{\text {lin }}(X)=1$.

Lemma 2.5. Suppose $K$ is the quotient field of a Henselian discrete valuation $\operatorname{ring} R$ with algebraically closed residue field $k$. We then have

(1) if $\operatorname{char}(k)=0$, then $\operatorname{ind}(X)$ divides $\operatorname{ind}_{\text {lin }}(X)$,

(2) if $\operatorname{char}(k)=p>0$, then the prime-to- $p$ part of $\operatorname{ind}(X)$ divides that of $\operatorname{ind}_{\text {lin }}(X)$,

Proof. The proof follows easily from [ELW15, Theorem 3.2]. 
Definition 2.6. Denote by $G$ the absolute Galois group $\operatorname{Gal}(\bar{K} / K)$. An invertible sheaf $\mathcal{L}_{\bar{K}}$ on $X_{\bar{K}}:=X \times_{K} \operatorname{Spec}(\bar{K})$ is called $G$-invariant if for any $\sigma \in G$ and the induced morphism $\sigma: X_{\bar{K}} \rightarrow X_{\bar{K}}$, we have $\sigma^{*} \mathcal{L}_{\bar{K}} \cong \mathcal{L}_{\bar{K}}$.

Theorem 2.7. Let $H$ be an ample divisor on $X$ such that if there exists an invertible sheaf $H_{0}$ on $X$ with $H_{0}^{\otimes n} \cong H$, then $n=1$ or -1 . Suppose that $H^{1}\left(\mathcal{O}_{X}\right)=0, \operatorname{Pic}\left(X_{\bar{K}}\right)$ is of rank $r$, generated by $\mathcal{L}_{1}, \ldots, \mathcal{L}_{r-1}$ and $\mathcal{L}_{r}:=H_{\bar{K}}=H \otimes_{K} \bar{K}$ satisfying the following conditions:

(1) the ideal $\left(\operatorname{deg}\left(\mathcal{L}_{1}\right), \operatorname{deg}\left(\mathcal{L}_{2}\right), \ldots, \operatorname{deg}\left(\mathcal{L}_{r}\right)\right)$ in $\mathbb{Z}$ generated by $\operatorname{deg}\left(\mathcal{L}_{i}\right)$ for $i=1, \ldots, r$ coincides with the ideal $(1)$, where $\operatorname{deg}\left(\mathcal{L}_{i}\right)$ is with respect to $H_{\bar{K}}$,

(2) for any $r \times r$-matrix $A=\left(a_{i, j}\right)$ with integral entries $a_{i, j}, a_{r, k}=0$ for all $k<r, a_{r, r}=1$, $A \neq \operatorname{Id}$ and $A^{t}=\operatorname{Id}$ for some $t>0$, we have $\sum_{j} a_{i j} \operatorname{deg}\left(\mathcal{L}_{j}\right) \neq \operatorname{deg}\left(\mathcal{L}_{i}\right)$ for some $i>0$.

Then, each $\mathcal{L}_{i}$ is $G$-invariant and $\operatorname{gcd}\left\{\chi\left(\mathcal{L}_{i}(n)\right) \mid i=1, \ldots, r\right.$ and $\left.n \in \mathbb{Z}\right\}=1$, where $\mathcal{L}_{i}(n):=$ $\mathcal{L}_{i} \otimes H_{\bar{K}}^{\otimes n}$.

Proof. By [Gro66, Théorème 8.5.2], there exists a finite field extension $K^{\prime}$ of $K$ and an invertible sheaf $\mathcal{L}_{i}^{\prime}$ on $X_{K^{\prime}}=X \times_{K} \operatorname{Spec}\left(K^{\prime}\right)$ such that $\mathcal{L}_{i} \cong \mathcal{L}_{i}^{\prime} \otimes_{K^{\prime}} \bar{K}$ i.e., the pull-back of $\mathcal{L}_{i}^{\prime}$ to $X_{\bar{K}}$ is isomorphic to $\mathcal{L}_{i}$. Without loss of generality (replace $K^{\prime}$ by the smallest Galois extension of $K$ containing $K^{\prime}$ ), we can assume that $K^{\prime}$ is a finite Galois extension of $K$. Denote by $\mathcal{L}_{r}^{\prime}:=H_{K^{\prime}}=H \otimes_{K} K^{\prime}$. Let $\sigma \in \operatorname{Gal}\left(K^{\prime} / K\right)$ and $\sigma: X_{K^{\prime}} \rightarrow X_{K^{\prime}}$ the induced morphism. Suppose that

$$
\sigma^{*} \mathcal{L}_{i}^{\prime} \cong \bigotimes_{j=1}^{r}\left(\mathcal{L}_{j}^{\prime}\right)^{\otimes a_{i, j}} \text { for some integer } a_{i, j}, i<r .
$$

As $H$ comes from $X$, we have $\sigma^{*} \mathcal{L}_{r}^{\prime} \cong \mathcal{L}_{r}^{\prime}$. Suppose that $\sigma^{*} \mathcal{L}_{i}^{\prime} \not \mathcal{L}_{i}^{\prime}$ for some $i>0$. Then there exists $j \neq i$ such that $a_{i, j} \neq 0$. In particular, the matrix $A:=\left(a_{i, j}\right)$ is not the identity matrix. Note that, $a_{r, j}=0$ for all $j<r$ and $a_{r, r}=1$. Since $\sigma$ is of finite order, there exists an integer $b$ such that

$$
\mathcal{L}_{i}^{\prime} \cong\left(\sigma^{*}\right)^{b}\left(\mathcal{L}_{i}^{\prime}\right)=\bigotimes_{j=1}^{r}\left(\mathcal{L}_{j}^{\prime}\right)^{\otimes b_{i, j}} \text { where } A^{b}=\left(b_{i, j}\right), i=1, \ldots, r, j=1, \ldots, r .
$$

In other words, $A^{b}=\mathrm{Id}$. Since the Hilbert function of $\mathcal{L}_{i}^{\prime}$ is the same as that of $\sigma^{*} \mathcal{L}_{i}^{\prime}$, we conclude that $\operatorname{deg}\left(\mathcal{L}_{i}^{\prime}\right)=\sum_{j} a_{i, j} \operatorname{deg}\left(\mathcal{L}_{j}^{\prime}\right)$. But, this contradicts our assumption $(2)$. Hence, $\sigma^{*} \mathcal{L}_{i}^{\prime} \cong \mathcal{L}_{i}^{\prime}$ for all $i=1, \ldots, r$. In other words, each $\mathcal{L}_{i}$ is $G$-invariant.

We now prove that $\operatorname{gcd}\left\{\chi\left(\mathcal{L}_{i}(n)\right) \mid i=1, \ldots, r\right.$ and $\left.n \in \mathbb{Z}\right\}=1$. Denote by $P_{i}(t)$ (resp. $\left.P_{0}(t)\right)$ the Hilbert polynomial of the invertible sheaf $\mathcal{L}_{i}\left(\operatorname{resp} . \mathcal{O}_{X_{\bar{K}}}\right)$ for $i=1, \ldots, r$. Note that the leading coefficient of $Q_{i}(t):=P_{i}(t)-P_{0}(t)$ is $\operatorname{deg}\left(\mathcal{L}_{i}\right) /(d-1)$ !, where $d=\operatorname{dim} X$ (see [HL10, Definition 1.2.11]). We claim that $\operatorname{gcd}\left\{Q_{i}(n) \mid n \in \mathbb{Z}\right\}$ divides $\operatorname{deg}\left(\mathcal{L}_{i}\right)$ for each $i=1, \ldots, r$. Indeed, denote by $D^{1} Q_{i}(t):=Q_{i}(t+1)-Q_{i}(t)$ and recursively, $D^{j} Q_{i}(t):=D^{j-1} Q_{i}(t+1)-D^{j-1} Q_{i}(t)$. Note that, $D^{j} Q_{i}(t)$ is of degree $d-1-j$ with leading coefficient $(d-1)(d-2) \ldots(d-j) \operatorname{deg}\left(\mathcal{L}_{i}\right) /(d-1)$ ! for all $j \geq 1$. Thus,

$$
D^{d-1} Q_{i}(t)=(d-1) ! \operatorname{deg}\left(\mathcal{L}_{i}\right) /(d-1) !=\operatorname{deg}\left(\mathcal{L}_{i}\right) .
$$

It follows immediately, $\operatorname{gcd}\left\{Q_{i}(n) \mid n \in \mathbb{Z}\right\}$ divides $\operatorname{deg}\left(\mathcal{L}_{i}\right)$. This proves the claim. Now, $\operatorname{gcd}\left\{\chi\left(\mathcal{L}_{i}(n)\right) \mid i=1, \ldots, r\right.$ and $\left.n \in \mathbb{Z}\right\}$ divides $\operatorname{gcd}\left\{\chi\left(\mathcal{L}_{i}(n)\right)-\chi\left(\mathcal{O}_{X_{\bar{K}}}(n)\right) \mid i=1, \ldots, r\right.$ and $\left.n \in \mathbb{Z}\right\}$ which is equal to $\operatorname{gcd}\left\{Q_{i}(n) \mid n \in \mathbb{Z}, i=1, \ldots, r\right\}$. Since the $\operatorname{gcd}\left\{Q_{i}(n) \mid n \in \mathbb{Z}, i=1, \ldots, r\right\}$ divides the generator of the ideal $\left(\operatorname{deg}\left(\mathcal{L}_{1}\right), \operatorname{deg}\left(\mathcal{L}_{2}\right), \ldots, \operatorname{deg}\left(\mathcal{L}_{r}\right)\right)=(1)$, we conclude that

$$
\operatorname{gcd}\left\{\chi\left(\mathcal{L}_{i}(n)\right) \mid i=1, \ldots, r \text { and } n \in \mathbb{Z}\right\}=1 .
$$


This proves the theorem.

Corollary 2.8. Suppose $K$ is the quotient field of a Henselian discrete valuation ring $R$ with algebraically closed residue field $k$. If $X$ satisfies the hypothesis of Theorem 2.7, then $\operatorname{ind}(X)=$ 1 if $\operatorname{char}(k)=0$ and prime-to-p part of ind $(X)$ equals 1 if $\operatorname{char}(k)=p>0$.

Proof. Recall the Brauer-Picard exact sequence:

$$
0 \rightarrow \operatorname{Pic}(X) \rightarrow \operatorname{Pic}\left(X_{\bar{K}}\right)^{G} \stackrel{\text { br }_{X}}{\longrightarrow} \operatorname{Br}(K) \rightarrow \operatorname{Br}(X) .
$$

Note that, in this case $\operatorname{Br}(K)=0$. Hence, every $G$-invariant invertible sheaf on $X_{\bar{K}}$ descends to an invertible sheaf on $X$. By Theorem 2.7, this implies

$$
\operatorname{ind}_{\operatorname{lin}}(X)=\operatorname{gcd}\left\{\chi\left(\mathcal{L}_{i}(n)\right) \mid i=1, \ldots, r \text { and } n \in \mathbb{Z}\right\}=1 .
$$

The corollary then follows directly from Lemma 2.5 .

The corollary gives numerous examples of smooth, projective varieties with index 1 .

Example 2.9. Suppose $K$ is the quotient field of a Henselian discrete valuation ring $R$ with algebraically closed residue field $k$. Let $X$ be a smooth, projective variety with $\operatorname{deg}\left(H_{\bar{K}}\right)>2$, $H^{1}\left(\mathcal{O}_{X}\right)=0, \operatorname{Pic}\left(X_{\bar{K}}\right)$ is of rank 2 and there exists an invertible sheaf $\mathcal{L}_{0}$ of degree coprime to $\operatorname{deg}\left(H_{\bar{K}}\right)$ (for example, see Remark 2.10 below). Theorem 2.7 implies that every invertible sheaf on $X_{\bar{K}}$ is $G$-invariant and Corollary 2.8 implies that

$$
\operatorname{ind}(X)=\operatorname{ind}_{\operatorname{lin}}(X)=1 .
$$

Indeed, we simply need to check that the two conditions in Theorem 2.7 are satisfied. Let $\mathcal{L}_{1}$ and $\mathcal{L}_{2}:=H_{\bar{K}}$ be the generators of $\operatorname{Pic}\left(X_{\bar{K}}\right)$. Since $\mathcal{L}_{0}$ is a linear combination of $\mathcal{L}_{1}$ and $\mathcal{L}_{2}$ and $\operatorname{gcd}\left(\operatorname{deg}\left(\mathcal{L}_{0}\right), \operatorname{deg}\left(H_{\bar{K}}\right)\right)=1$, we have $\operatorname{gcd}\left(\operatorname{deg}\left(\mathcal{L}_{1}\right), \operatorname{deg}\left(H_{\bar{K}}\right)\right)=1$. In other words, the ideal $\left(\operatorname{deg}\left(\mathcal{L}_{1}\right), \operatorname{deg}\left(\mathcal{L}_{2}\right)\right)=1$ i.e., condition (1) of Theorem 2.7 is satisfied. Let

$$
A=\left(\begin{array}{cc}
a_{0} & a_{1} \\
0 & 1
\end{array}\right)
$$

be a matrix with integral entries. Note that, for any integer $b>0$

$$
A^{b}=\left(\begin{array}{cc}
a_{0}^{b} & a_{1}\left(a_{0}^{b-1}+a_{0}^{b-2}+\ldots+1\right) \\
0 & 1
\end{array}\right)
$$

Then, $A^{b}=\mathrm{Id}$ if and only if $a_{0}^{b}-1=0=a_{1}\left(a_{0}^{b-1}+a_{0}^{b-2}+\ldots+1\right)$. If $a_{1} \neq 0$ then $a_{0}=-1$. Since $\operatorname{deg}\left(\mathcal{L}_{2}\right)>2$ is coprime to $\operatorname{deg}\left(\mathcal{L}_{1}\right)$, we have $2 \operatorname{deg}\left(\mathcal{L}_{1}\right) \neq a_{1} \operatorname{deg}\left(\mathcal{L}_{2}\right)$ for any integer $a_{1}$. Thus condition (2) of Theorem 2.7 is satisfied. Hence, by Theorem 2.7 and Corollary 2.8, we conclude that $\mathcal{L}_{1}$ is $G$-invariant and $\operatorname{ind}(X)=\operatorname{ind}_{\text {lin }}(X)=1$.

Remark 2.10. Fix coordinates $X_{0}, X_{1}, X_{2}, X_{3}$ on $\mathbb{P}_{\mathbb{Q}}^{3}$. Take any $d \geq 4$ and $F_{1}, F_{2}$ two homogeneous polynomials of degree $d$ in variables $X_{i}$ and coefficients in $\mathbb{Q}$. It is easy to check that for general $F_{1}, F_{2}$, the surface defined by $F:=F_{1} X_{1}+F_{2} X_{2}$ is smooth and $\operatorname{rk}(\operatorname{Pic}(X))=2$ (the hyperplane section and the line defined by $X_{1}, X_{2}$ generate $\operatorname{Pic}(X)$ ). For any prime $p$, take $K=\mathbb{Q}_{p}^{u r}$, the maximal unramified extension of $\mathbb{Q}_{p}$. Note that $K$ is a $C_{1}$-field, hence $\operatorname{Br}(K)=0$. Let $X$ be the surface in $\mathbb{P}_{K}^{3}$ defined by $F$. By [Har10, Ex. III.5.5], we have $H^{1}\left(\mathcal{O}_{X}\right)=0$. Then Example 2.9 implies that $\operatorname{ind}_{\operatorname{lin}}(X)=1=\operatorname{ind}(X)$. One can similarly construct numerous examples of surfaces in $\mathbb{P}_{K}^{3}$ of any degree, satisfying the conditions of Theorem 2.7, arising from the theory of Noether-Lefschetz locus (see [Voi88, Voi89]), thereby having index 1.

We now give some examples in the case $\operatorname{Br}(K) \neq 0$, in particular $K$ is not a $C_{1}$-field.

Example 2.11. Let $K=\mathbb{R}$ and $G:=\operatorname{Gal}(\bar{K} / K)$ the absolute Galois group. Recall, $\operatorname{Br}(\mathbb{R})=$ $\mathbb{Z} / 2 \mathbb{Z}$. Denote by $\operatorname{br}_{X}: \operatorname{Pic}\left(X_{\bar{K}}\right)^{G} \rightarrow \operatorname{Br}(\mathbb{R})$ as in the Brauer-Picard exact sequence (2.1). 
(1) We first consider the case, when $\operatorname{br}_{X}$ is the zero map. Let $X$ be the smooth, projective surface in $\mathbb{P}_{\mathbb{R}}^{3}$ defined by the equation $X_{0}^{2}+X_{1}^{2}+X_{2}^{2}-X_{3}^{2}=0$, where $X_{i}$ are the coordinates of $\mathbb{P}^{3}$ for $i=0, \ldots, 3$. Note that, $X_{\mathbb{C}}:=X \times_{\mathbb{R}} \operatorname{Spec}(\mathbb{C})$ contains the two lines $L_{1}:=Z\left(X_{0}-i X_{1}, X_{2}-X_{3}\right)$ and $L_{2}:=Z\left(X_{0}+i X_{1}, X_{2}-X_{3}\right)$. The element of the absolute Galois group $G$ sending $i$ to $-i$ interchanges $L_{1}$ and $L_{2}$. Since $\operatorname{Pic}\left(X_{\mathbb{C}}\right)=\mathbb{Z}^{\oplus 2}$, generated by $L_{1}$ and $L_{2}$, we conclude that the $G$-invariant subgroup of $\operatorname{Pic}\left(X_{\mathbb{C}}\right)$ is generated as a $\mathbb{Z}$ module by $L_{1}+L_{2}$, which is linearly equivalent to the hyperplane section $H_{\mathbb{C}}:=H \otimes_{\mathbb{R}} \mathbb{C}$. Therefore, in this case $\operatorname{Pic}\left(X_{\mathbb{C}}\right)^{G}$ consists of points corresponding to multiples of the invertible sheaf $H_{\mathbb{C}}$. Since $H_{\mathbb{C}}$ comes from $X$, the exactness of (2.1) implies that br $_{X}$ is the zero map. It is easy to check that $\operatorname{ind}_{\text {lin }}(X)=1$ (Euler characteristic of $\mathcal{O}_{X}$ is 1 ). Note that, $\operatorname{ind}(X)=1$ as $X$ contains $\mathbb{R}$-rational points.

(2) We now consider the case, when $\mathrm{br}_{X}$ is non-trivial (equivalently surjective). Let $X$ be the $\mathbb{R}$-plane conic defined by $X_{0}^{2}+X_{1}^{2}+X_{2}^{2}=0$, where $X_{i}$ are the coordinates of $\mathbb{P}_{\mathbb{R}}^{2}$, for $i=0,1,2$. In this case, $h^{1}\left(\mathcal{O}_{X}\right)=0$, i.e., $\operatorname{Pic}^{0}(X)=0$. This implies, there exists an unique invertible sheaf $\mathcal{L}_{\bar{K}}$ on $X_{\bar{K}}$ of degree 1 , hence it is $G$-invariant. If $\operatorname{br}_{X}\left(\mathcal{L}_{\bar{K}}\right)=0$, then by the exact sequence (2.1) there exists an invertible sheaf $\mathcal{L}$ on $X$ such that $\mathcal{L}_{\bar{K}} \cong \mathcal{L} \otimes_{K} \bar{K}$. Since $\operatorname{deg}(\mathcal{L})=1$, the Riemann-Roch theorem would then imply that $X$ contains a rational point, which gives us a contradiction. Hence, $b_{X}$ is non-trivial. Using [Kol13, (1.3)], we have $\operatorname{ind}_{\text {lin }}(X)$ is the ged of $\operatorname{ind}(X)$ and $1-\rho_{a}(X)$. Since $\operatorname{ind}(X)=2$, we have $\operatorname{ind}_{\text {lin }}(X)=\operatorname{ind}(X)=2$ (observe $\left.\rho_{a}(X)=1\right)$.

\section{REFERENCES}

[BFM75] P. Baum, W. Fulton, and R. MacPherson. Riemann-roch for singular varieties. Publications Mathématiques de l'IHÉS, 45:101-145, 1975.

[ELW15] H. Esnault, M. Levine, and O. Wittenberg. Index of varieties over Henselian fields and Euler characteristic of coherent sheaves. Journal of Algebraic Geometry, 24(4):693-718, 2015.

[Gro66] A. Grothendieck. Éléments de géométrie algébrique (rédigés avec la collaboration de Jean Dieudonné): IV. étude locale des schémas et des morphismes de schémas, troisième partie. Publications Mathématiques de l'IHÉS, 28:5-255, 1966.

[Har10] R. Hartshorne. Deformation Theory. Graduate text in Mathematics. Springer-Verlag, 2010.

[HL10] D. Huybrechts and M. Lehn. The geometry of moduli spaces of sheaves. Springer, 2010.

[Kau16] I. Kaur. The $C_{1}$ conjecture for the moduli space of stable vector bundles with fixed determinant on a smooth projective curve. Ph. d. thesis, Freie University Berlin, 2016.

[Kau18] I. Kaur. A pathological case of the $C_{1}$ conjecture in mixed characteristic. Mathematical Proceedings of the Cambridge Philosophical society, 2018.

[Kol13] J. Kollár. Esnault-Levine-Wittenberg indices. arXiv preprint arXiv:1312.3923, 2013.

[Lan52] S. Lang. On quasi algebraic closure. Annals of Mathematics, pages 373-390, 1952.

[Ser13] J. P. Serre. Local fields, volume 67. Springer Science \& Business Media, 2013.

[Voi88] C. Voisin. Une précision concernant le théorème de Noether. Math. Ann., 280(4):605-611, 1988.

[Voi89] C. Voisin. Composantes de petite codimension du lieu de Noether-Lefschetz. Comm. Math. Helve., 64(4):515-526, 1989. SPAIN

BCAM - Basque Centre for Applied Mathematics, Alameda de Mazarredo 14, 48009 Bilbao,

E-mail address: adan@bcamath.org

Instituto de Matemática Pura e Aplicada, Estr. Dona Castorina, 110 - Jardim Botânico, Rio DE JANEIRO - RJ, 22460-320, BRAZIL

E-mail address: inder@impa.br 